\title{
Lockdown, Stress und Leistungsdruck
}

\author{
Chronischer Kopfschmerz bei Kindern und Jugendlichen war eines jener Themen, die im Rahmen der \\ Pressekonferenz zum diesjährigen Deutschen Schmerzkongress diskutiert wurden. Ein weiterer Beitrag \\ erörterte mögliche COVID-bedingte Ursachen für anhaltende Kopfschmerzen.
}

n einer Querschnittsstudie in Dresden mit über 2.700 befragten Schüler*innen gaben mehr als zwei Drittel aller Befragten an, regelmäßig an Kopfschmerzen zu leiden.' Mehr als ein Fünftel aller Kinder und Jugendlichen mit mehr als zwei Kopfschmerztagen im Monat fehlte dadurch regelmäßig in der Schule. Oftmals sind Leistungsdruck, emotionaler Stress, zu viel Zeit am Bildschirm und zu wenig Bewegung die Ursache - der monatelange Lockdown hat all diese Faktoren noch einmal deutlich verstärkt.

Wenn Kinder und Jugendliche regelmäBig an Kopfschmerzen leiden, können sie schnell in einen Teufelskreis aus Leistungsabfall, Schulangst und sozialer Isolation geraten. ${ }^{2}$ Dennoch werden Kopfschmerzen bei Kindern häufig nicht ernst genommen. „Eine ärztliche Diagnose und Therapie der Kopfschmerzen erhalten nur die wenigsten“, sagt PD Dr. Gudrun Goßrau, Leiterin der Kopfschmerzambulanz im Interdisziplinären Universitätsschmerzzentrum am Universitätsklinikum Dresden und Kongresspräsidentin. „Eltern sollten Kopfschmerzen nicht bagatellisieren. Kopfschmerzen können den Alltag und die Zukunft junger Menschen stark beeinträchtigen." Alarmierend sei, dass Kopfschmerzen stattdessen häufig in Eigenregie mit frei verkäuflichen Medikamenten bekämpft würden. „Schmerzmittel sollten Kinder aber nur einnehmen, wenn sie von Ärzt*innen in geeigneter Dosierung verordnet wurden", so Dr. Goßrau. Denn bei häufiger Einnahme könnten Medikamente die Kopfschmerzen auch verstärken. Manche seien für Kinder gar nicht geeignet.

Daten einer aktuellen Münchner Studie zeigen, dass Migräne in der vulnerablen Übergangsphase zwischen Jugend- und Erwachsenenalter mit einem erhöhten Risiko für die Entwicklung weiterer Schmerzen im Erwachsenenalter assoziiert ist. ${ }^{3}$ „Es besteht deshalb akuter Handlungsbedarf, wenn Kinder regelmäßig an Kopfschmerzen leiden“, sagt Dr. Goßrau. Haben Kopfschmerzen bei Kindern und Jugendlichen erst einmal Einschränkungen des Alltags zur Folge, bedarf es interdisziplinärer Kon-

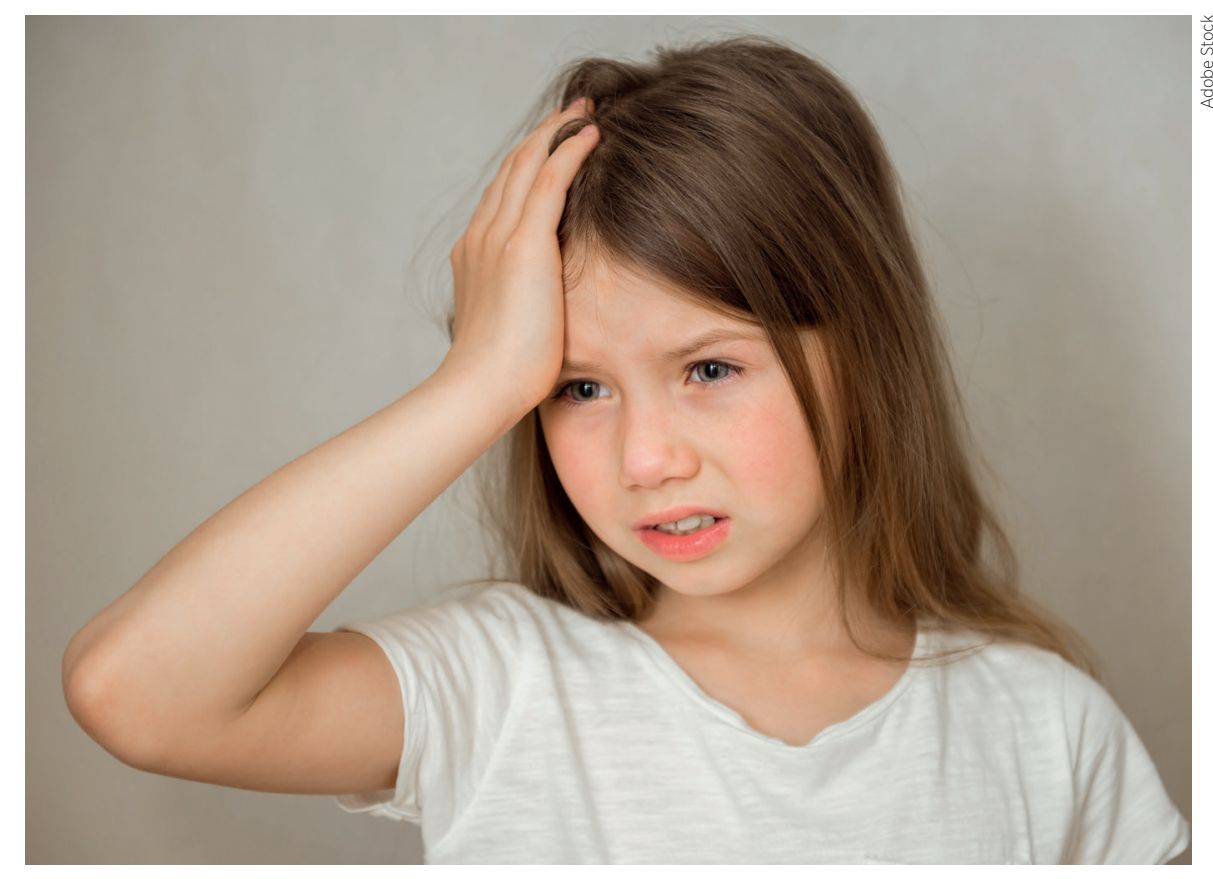

zepte - wie etwa des Dresdner Kinderkopfschmerzprogramms (DreKiP). Es handelt sich um ein Gruppentherapieprogramm, das aus acht unterschiedlichen Modulen besteht.

„In Deutschland besteht nach wie vor ein Versorgungsbedarf, der mit den vorhandenen Therapiestrukturen nicht abgedeckt wird“", so Dr. Goßrau. Es brauche daher eine Sensibilisierung von Eltern und Lehrenden, eine Berücksichtigung von Kopfschmerzen als Krankheitssymptom sowie eine entsprechende Ausbildung der Akteur*innen im Gesundheitssystem. „Darüber hinaus müssten dringend spezifische wie auch interdisziplinäre Therapiemöglichkeiten für Kinder und Jugendliche mit Kopfschmerzen geschaffen werden.“

\section{COVID-19: MÖGLICHE URSACHEN FÜR ANHALTENDE KOPFSCHMERZEN}

„Die COVID-19-Pandemie hat auf mehreren Ebenen Auswirkungen auf Schmerzen beziehungsweise auf die Behandlung von Schmerzpatient*innen“, sagt Professor Dr. Winfried Meißner, Leiter der Sektion Schmerztherapie in der Klinik für Anästhesiologie und Intensivmedizin am Universitätsklinikum Jena und Präsident der
Deutschen Schmerzgesellschaft. Neben den akuten Schmerzen, die als direkte Krankheitsfolge auftreten, kommt es bei einem Teil der Patient*innen auch zu lang anhaltenden Beschwerden, die durch die Krankheit selbst oder durch die intensivmedizinische Behandlung verursacht werden. „Diese Phänomene sind zwar inzwischen gut beschrieben und zahlenmäßig erfasst - die Mechanismen, auf denen sie beruhen, sind jedoch noch weitgehend unklar", sagt Professor Dr. Andreas Straube, Oberarzt an der Neurologischen Klinik und Poliklinik der LMU München. Der Kopfschmerzexperte verweist dabei auf Studien, die zeigen, dass Betroffene, die schon vorher an einem primären Kopfschmerz, meist Migräne, erkrankt waren, nach einer SARS-CoV-2-Infektion über eine Verstärkung dieser Kopfschmerzen berichten. Andere Patient*innen entwickelten einen neuen, bisher nicht bekannten, anhaltenden Kopfschmerz. ${ }^{4}$

Für die akuten Schmerzen könnten sowohl neuropathische, also auf einer Schädigung oder Fehlfunktion von Nerven beruhende, als auch entzündliche Mechanismen verantwortlich sein. Bei den chronischen Formen wird in neueren Forschungsarbeiten eine Beteiligung des Inflammasoms 
diskutiert. ${ }^{4}$ „Dabei handelt es sich um einen Eiweißkomplex, der sich innerhalb von Zellen befindet und als Reaktion auf Krankheitserreger oder zellulären Stress aktiviert wird“, erläutert Prof. Straube. Als Teil der angeborenen Immunabwehr ist das Inflammasom in der Lage, die Freisetzung von Entzündungsbotenstoffen zu veranlassen - ein Mechanismus, der möglicherweise nicht nur bei der Entstehung von lang anhaltenden Kopfschmerzen nach COVID-19 eine Rolle spielt, sondern auch bei der Chronifizierung von primären - also ohne erkennbare Ursache auftretenden - Kopfschmerzen wie der Migräne. „Es spricht einiges dafür, dass diese Kopfschmerzformen auf dieselben Mechanismen zurückzuführen sind“, sagt Prof. Straube und äußert die Hoffnung, dass sich aus der Erforschung COVID-bedingter Kopfschmerzen neue Behandlungsansätze ergeben, die dann auch Menschen mit den bisher nur schlecht therapierbaren primären Kopfschmerzen zugutekommen.

Lang anhaltende Schmerzen und Muskelschwäche können auch als Folge der intensivmedizinischen Behandlung auftreten - ein Phänomen, das als Critical illness neuropathy/myopathy (CINM) bezeichnet wird und auf die Schädigung und Fehlfunktion einzelner Nerven zurückgeht. „Die CINM ist in der Intensivmedizin lange bekannt", sagt Prof. Meißner. Die Beschwerden seien jedoch bei COVID-19-Betroffenen deutlich häufiger als bei anderen Patientengruppen. In einer schwedischen Studie war mindestens jeder/jede sechste COVID-19Patient*in nach einer intensivmedizinischen Betreuung von CINM betroffen - mit teils stark einschränkenden Folgen. ${ }^{5}$

Referenzen:

Redaktion

1 Nieswand $\vee$ et al. Cephalalgia. 2019 Jul; 39(8): 1030-1040

2 Nieswand $V$ et al. doi link: https://doi.org/10.1007/ S11916-020-00892-6; published: 2020-10

3 Gerstl L et al. Cephalalgia. 2021 Jun; 23:3331024211021792; doi: 10.1177/03331024211021792

4 Caronna E et al. Cephalalgia. 2020; 40(13):14101421; doi: 10.1177/0333102420965157

5 Frithiof R et al. Clin Neurophysiol. 2021 Jul; 132(7): 1733-1740; doi: 10.1016/j.clinph.2021.03.016. Epub 2021 Apr 1

Quelle: Pressegespräch zur Eröffnung der Jahrestagung der Deutschen Schmerzgesellschaft e. V. und der Deutschen Migräne- und Kopfschmerzgesellschaft (DMKG) e. V., 20. Oktober 2021, Mannheim (hybrid)

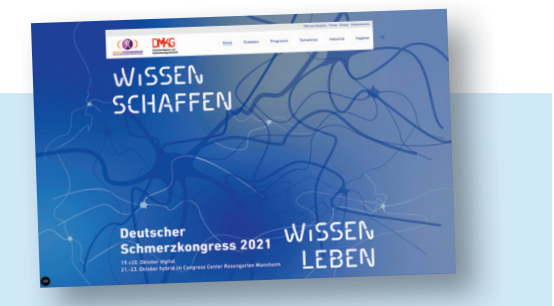

DEUTSCHER SCHMERZKONGRESS

Zum ersten Mal wurde der gemeinsame Kongress der Deutschen Schmerzgesellschaft e. V. und der Deutschen Migräneund Kopfschmerzgesellschaft (DMKG) e. V. als hybride Veranstaltung abgehalten. Die Jahrestagung fand am 19. und 20. Oktober 2021 digital, von 21. bis 23. Oktober 2021 dann hybrid in Mannheim statt. Über 1.000 Personen waren vor Ort, rund 600 schalteten sich zudem von ihren heimischen Rechnern dazu.

Der Schmerzkongress stand in diesem Jahr unter dem Motto „Wissen schaffen - Wissen leben“. Zu den Schwerpunktthemen gehörten unter anderem „Kopfschmerzen bei Kindern und Jugendlichen“ sowie „COVID-19-assoziierte Schmerzsyndrome“. Die Sitzungen stehen auf der Online-Plattform noch ein Jahr lang zur Verfügung.

\section{KONGRESSKALENDER*}

\section{FEBRUAR 2022}

\section{Endoprothetikkongress}

10.-12. Februar, Berlin

Information und Anmeldung:

endokongress.de

\section{MÄRZ 2022}

\section{Dattelner Kinderschmerztage}

10.-12. März, Recklinghausen Information und Anmeldung: kinderpalliativzentrum.de/weiterbildung/dattelner-kinderschmerztage

\section{ÖGN 2022}

16.-18. März, Graz

Information und Anmeldung:

oegn-jahrestagung.at

\section{Deutscher Schmerz- und Palliativtag}

22.-26. März, online

Information und Anmeldung:

dgschmerzmedizin.de

\section{WCO-IOF-ESCEO 2022}

24.-27. März, Berlin

Information und Anmeldung:

wco-iof-esceo.org

\section{APRIL 2022}

\section{Schmerzkongress}

22. April, Moorheilbad Harbach

(Workshop 21. April 2022)

Information und Anmeldung:

moorheilbad-harbach.at/

schmerzkongress

\section{EFIC 2022}

27.-30. April, Dublin

Information und Anmeldung:

efic-congress.org

\section{MAI 2022}

\section{Dreiländertagung Kopfschmerz \\ 12.-14. Mai, Lindau \\ Information und Anmeldung: \\ dreilaendertagung-kopfschmerz.de}

\section{JUNI 2022}

\section{EULAR 2022}

1.-4. Juni, Kopenhagen/online Information und Anmeldung: congress.eular.org

\section{BVSD-Kongress}

24.-25. Juni, Berlin

Information und Anmeldung:

bvsd.de/kongresse

\section{EAN 2022}

25.-28. Juni, Wien

Information und Anmeldung:

ean.org/congress2022

* Planungsstand 15. 11.2021. Aufgrund der COVID-19-Regelungen sind kurzfristige Änderungen möglich Bitte erkundigen Sie sich bei dem jeweiligen Veranstalter, ob die Veranstaltung planmäßig stattfindet. 